Forschende

Komplementärmedizin und Klassische Naturheilkunde

\section{Tagungen und Kongresse - Meetings and Conferences}

Forsch Komplementärmed Klass Naturheilkd 2003;10:355

\subsection{2.-03.03.2004}

Freudenstadt

Deutschland

\subsection{3.-04.04.2004 \\ Bad Wildungen}

Deutschland

17.04.-21.04.2004

Wiesbaden

Deutschland

\title{
25.06.-04.07.2004
}

Schönau a. Königsee

Deutschland

29.08.-03.09.2004

Pörtschach

Österreich

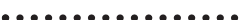

30.09.-06.10.2004

Freudenstadt

Deutschland

\subsubsection{4}

Baden

Schweiz

106. Kongress des Zentralverbandes der Ärzte für Naturheilverfahren und

Leitthema: Energie und Information in der Medizin Regulationsmedizin e.V. - ZÄN

\section{Naturheilverfahren - Kurs C und D}

107. Kongress des Zentralverbandes der Ärzte für Naturheilverfahren und Regulationsmedizin e.V. - ZÄN

Leitthema: Die Frau in der Medizin

19. Schweizerische Tagung für Phytotherapie - Evaluation Phytotherapie eine Bilanz
Auskunft: Geschäftsstelle des ZÄN

Am Promenadenplatz 1

D-72250 Freudenstadt

Tel. +49 744191 858-0, Fax -22

E-mail ZAEN-Freudenstadt@t-online.de

www.zaen.org

Auskunft: Berufsverband Deutscher Internisten e.V.

Kongressabteilung

Postfach 1566

D-65005 Wiesbaden

Tel. +49 611 18133-22 oder -24, Fax -23

E-mail info@bdi.de

www.bdi.de

-..........

Auskunft: Berufsverband Deutscher Internisten e.V.

Kongressabteilung

Postfach 1566

D-65005 Wiesbaden

Tel. +49 611 18133-22 oder -24, Fax -23

E-mail info@bdi.de

www.bdi.de

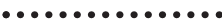

Auskunft: Berufsverband Deutscher Internisten e.V.

Kongressabteilung

Postfach 1566

D-65005 Wiesbaden

Tel. +49 611 18133-22 oder -24, Fax -23

E-mail info@bdi.de

www.bdi.de

53. Seminarkongress - Schwerpunkt Rheumatologie, Infektionskrankheiten und klinische Pharmakologie

Auskunft: Berufsverband Deutscher Internisten e.V.

Kongressabteilung

Postfach 1566

D-65005 Wiesbaden

Tel. +49 611 18133-22 oder -24, Fax -23

E-mail info@bdi.de

www.bdi.de

Auskunft: Geschäftsstelle des ZÄN

Am Promenadenplatz

D-72250 Freudenstadt

Tel. +49 744191 858-0, Fax -22

E-mail ZAEN-Freudenstadt@t-online.de

www.zaen.org

Auskunft: Sekretariat SMGP

Keltenstr. 40

CH-8044 Zürich

Tel. +41 125 21-879, Fax -23

E-mail sekretariat-smgp@swissonline.ch

www.smgp.ch 